\title{
Simulations of Lateral Transport and Dropout Structure of Energetic Particles from Impulsive Solar Flares
}

\author{
P. Tooprakai ${ }^{a, b}$, A. Seripienlert ${ }^{b, c, d, e}$, D. Ruffolo ${ }^{* b, c}$, P. Chuychai ${ }^{b, f}$, W. H. Matthaeus ${ }^{g}$ \\ ${ }^{a}$ Department of Physics, Faculty of Science, Chulalongkorn University, Bangkok 10330, Thailand. \\ ${ }^{b}$ Thailand Center of Excellence in Physics, CHE, Ministry of Education, Bangkok 10400, \\ Thailand. \\ ${ }^{c}$ Department of Physics, Faculty of Science, Mahidol University, Bangkok 10400, Thailand. \\ ${ }^{d}$ Division of Physics, Faculty of Science and Technology, Rajamangala University of Technology \\ Thanyaburi, Pathum Thani 12110, Thailand. \\ ${ }^{e}$ Now at Dacon Inspection Services Co., Ltd., Rayong 21130, Thailand. \\ ${ }^{f}$ Department of Physics, Faculty of Science, Burapha University, Chonburi 20131, Thailand. \\ ${ }^{g}$ Bartol Research Institute and Department of Physics and Astronomy, University of Delaware, \\ Newark, DE 19716, USA. \\ E-mail: paisan.to@chula.ac.th, tikim7@gmail.com, \\ david.ruf@mahidol.ac.th, piyanate@gmail.com, whm@udel.edu.
}

\begin{abstract}
We simulate trajectories of energetic particles from impulsive solar flares for 2D+slab models of magnetic turbulence in spherical geometry to study dropout features, i.e., sharp, repeated changes in the particle density. Among random-phase realizations of two-dimensional (2D) turbulence, a spherical harmonic expansion can generate homogeneous turbulence over a sphere, but a $2 \mathrm{D}$ fast Fourier transform (FFT) locally mapped onto the lateral coordinates in the region of interest is much faster computationally, and we show that the results are qualitatively similar. We then use the 2D FFT field as input to a 2D MHD simulation, which dynamically generates realistic features of turbulence such as coherent structures. The magnetic field lines and particles spread nondiffusively (ballistically) to a patchy distribution reaching up to 25 degrees from the injection longitude and latitude at $r \sim 1 \mathrm{AU}$. This dropout pattern in field line trajectories has sharper features in the case of the more realistic 2D MHD model, in better qualitative agreement with observations. The initial dropout pattern in particle trajectories is relatively insensitive to particle energy, though the energy affects the pattern's evolution with time. We make predictions for future observations of solar particles near the Sun (e.g., at $0.25 \mathrm{AU}$ ), for which we expect a sharp pulse of outgoing particles along the dropout pattern, followed by backscattering that first remains close to the dropout pattern and later exhibits cross-field transport to a distribution that is more diffusive, yet mostly contained within the dropout pattern found at greater distances.
\end{abstract}

35th International Cosmic Ray Conference

12-20 July, 2017

Bexco, Busan, Korea

\footnotetext{
* Speaker.
} 


\section{Introduction}

Observations of energetic particles from impulsive solar flares, which have narrow source regions of particle injection at the Sun [19], can exhibit "dropouts" in which the particle flux at a spacecraft disappears and reappears repeatedly $[10,15]$. This has been interpreted in terms of a filamentary distribution of magnetic field lines connected to the source region, which convects past the spacecraft along with the solar wind. The filamentation has been attributed to photospheric motions [9] or in situ interplanetary turbulence [21, 28].

In our work (see also [26]), we model the transport of SEPs from impulsive solar flares using the 2D+slab model of magnetic turbulence, which has been shown to provide a useful model of observed solar wind fluctuations [3, 13] and parallel scattering of energetic particles [2], and has shown to be equivalent to a model of fluctuations generated by photospheric motions [8]. Previous simulations in Cartesian geometry have demonstrated that dropout patterns naturally result from magnetic field line trajectories in 2D+slab turbulence [21], due to temporary topological trapping of some trajectories near O-points of the 2D turbulence with suppressed diffusive escape [6], a mechanism that also applies to particle trajectories [25]. The combination of these mechanisms can lead to local trapping boundaries [5, 23], which may have been found by in situ observations in the solar wind (e.g., $[24,27])$. Here we use a spherical geometry, for different types of 2D turbulence models together with slab fluctuations, and trace trajectories of magnetic field lines and protons of varying energy as a function of distance from the Sun.

\section{Modeling}

\subsection{D+Slab Magnetic Turbulence Models in Spherical Geometry}

In spherical geometry, we express the total magnetic field as [22]

$$
\mathbf{B}=\mathbf{B}_{0}(r)+\mathbf{b}(\mathbf{r})=\frac{r_{1}^{2}}{r^{2}}\left[B_{1} \hat{\mathbf{r}}+\mathbf{b}^{\text {slab }}(r)+\mathbf{b}^{2 D}(\varphi, \Lambda)\right] .
$$

Here $\mathbf{B}_{0}$ is a radial mean magnetic field of magnitude $B_{1}$ at $r_{1}$, which we take to be 1 AU. The two fluctuation components, the slab turbulence $\mathbf{b}^{\text {slab }}$ and $2 \mathrm{D}$ turbulence $\mathbf{b}^{2 D}$, are perpendicular to the mean field, which is consistent with observations that magnetic turbulence in the solar wind is $\approx$ $90 \%$ transverse to the mean field $[1,12]$. The $2 \mathrm{D}$ field can be written as $\mathbf{b}^{2 D}(\varphi, \Lambda)=\nabla \times[a(\varphi, \Lambda) \hat{\mathbf{r}}]$, where $a(\varphi, \Lambda)$ is the magnetic potential function. We use the radial mean field as a rough approximation to the Parker spiral field [17] because it is difficult to formulate a global, homogeneous 2D fluctuating field for the latter. The fraction of fluctuation energy in slab fluctuations is taken to be $20 \%$, according to $[2,3]$. The slab fluctuations are first generated in Cartesian coordinates [26]. For a spherical geometry, we identify the parallel $z$-coordinate with the radius $r$ and use $b_{\varphi}^{\text {slab }}(r)=b_{x}^{\text {slab }}(z)$ and $b_{\Lambda}^{\text {slab }}(r)=b_{y}^{\text {slab }}(z) \sec \Lambda$, so that $\nabla \cdot \mathbf{b}^{\text {slab }}=0$. To some degree this violates the condition of homogeneity, but because we limit our simulations to an angular region of $-25^{\circ}<\varphi<25^{\circ}$ and $-25^{\circ}<\Lambda<25^{\circ}$, centered around the solar equator, the factor of $\sec \Lambda$ has only a minor effect.

For the 2D fluctuation component, we employed three different computational models; for details see [26]. The first model is a spherical harmonic expansion (SHE) of the potential function, evaluated up to $l_{\max }=2200$ using the numerical method of [11]. The second is a 2D FFT 
model, following [22], where we generate a potential function $a$ in terms of the Cartesian coordinates $(x, y)$ and then map it onto the angular coordinates $(\varphi, \Lambda)$ at the reference radius $r_{1}=1 \mathrm{AU}$. After generating $a\left(k_{x}, k_{y}\right)$, we use an inverse fast Fourier transform to efficiently calculate $a(x, y)$ at $1024 \times 1024$ grid points, and then map the Cartesian potential $a(x, y)$ onto the heliolongitude using $\varphi=x / r_{1}$ and the heliolatitude using $\Lambda=y / r_{1}$.

For an improved representation of 2D magnetic turbulence in the solar wind, our third method used the 2D FFT potential function in Cartesian coordinates as an initial condition and evolved the field for one nonlinear time according to the 2D MHD equations [23]. This leads to smoother equipotential contours and also tends to produce current sheets and other coherent structures that are not expected in random-phase representations such as the 2D SHE and 2D FFT models.

\subsection{Trajectories of Magnetic Field Lines and Particles}

We find the trajectories of magnetic field lines by using the streamline equation:

$$
\frac{d r}{B_{r}}=\frac{r d \Lambda}{B_{\Lambda}}=\frac{r \cos \Lambda d \varphi}{B_{\varphi}}
$$

which can be written in terms of components as

$$
\begin{aligned}
& \frac{d \Lambda}{d r}=\frac{1}{r} \frac{B_{\Lambda}}{B_{r}}, \\
& \frac{d \varphi}{d r}=\frac{1}{r \cos \Lambda} \frac{B_{\varphi}}{B_{r}} .
\end{aligned}
$$

For particle trajectories, we solve the Newton-Lorentz equation:

$$
\gamma m \dot{\mathbf{v}}=q(\mathbf{v} \times \mathbf{B}),
$$

where $\gamma=1 / \sqrt{1-v^{2} / c^{2}}$ is the Lorentz factor, $m$ is the particle mass, $\mathbf{v}$ is the particle velocity, $\mathbf{B}$ is the interplanetary magnetic field, $c$ is the speed of light, and $q$ is the particle charge.

The field line equations or Newton-Lorentz equation was solved by the Cash-Karp method [4, 18], a fifth-order Runge-Kutta method with an adaptive step size, using a version of the Streamline code [7]. In each case, 50,000 magnetic field line or particle trajectories were traced from random initial locations within a circle of angular radius $2.5^{\circ}$ at $r=0.1 \mathrm{AU}$. This serves to model the narrow injection region expected for an impulsive solar flare. The initial direction of each particle (at $t=0$ and $r=0.1 \mathrm{AU}$ ) was parallel to the local magnetic field line, due to the expected effect of strong adiabatic focusing at $r<0.1 \mathrm{AU}[20]$.

\section{Magnetic Connectivity to a Narrow Injection Region}

Figure 1 shows the angular coordinates of simulated magnetic field lines at varying radius $r$ from the Sun for MHD. After an initial concentration in a narrow injection region at $r=0.1 \mathrm{AU}$, the field line distribution distorts into a complex pattern at higher $r$, i.e., the initial flux tube "shreds" [14]. In all cases, we find that SEP trajectories closely follow these magnetic field line trajectories at early times. Then as an observer (spacecraft) moves relative to this pattern, the complex filamentation of magnetic connectivity leads to a "dropout" pattern of repeated disappearance and 

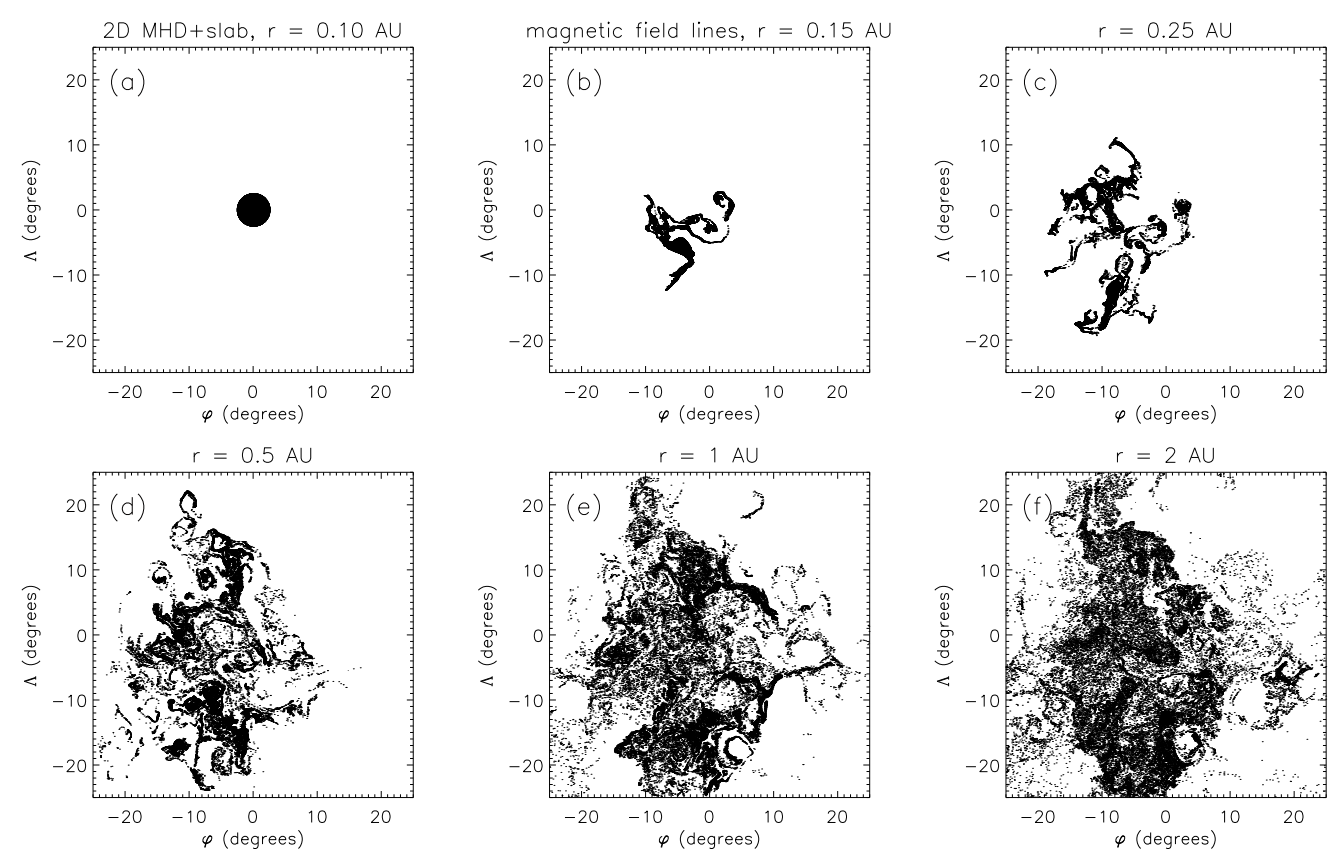

Figure 1: Trajectories of 50,000 magnetic field lines in heliolongitude and heliolatitude as a function of radius $r$ from the Sun, starting at random initial locations at $r=0.1 \mathrm{AU}$ within a circle of radius $2.5^{\circ}$, for the 2D MHD + slab magnetic fluctuation model. This model is a refinement of the random-phase 2D FFT model, which is used as the initial condition for solving the 2D MHD equations. The resulting 2D MHD magnetic field includes coherent structures, and the field line trajectories displayed here exhibit finer filamentation than for the random-phase field models.

reappearance of energetic particle flux. Therefore, we first discuss the fundamental issue of magnetic connectivity, which determines whether or not particles will have access to a given location at early times.

Comparing results for the two random-phase 2D magnetic fields, the dropout patterns are qualitatively similar for 2D SHE and 2D FFT [26]. This justifies the use of the computationally cheaper 2D FFT model for dropout modeling, and also its use as an initial condition for the 2D MHD procedure. While the dropout patterns for 2D MHD (Figure 1) are similar to those for 2D FFT on the largest scales, on smaller scales the 2D MHD model yields much finer (narrower) dropout features. This highlights the important role played by the coherent structures generated by the MHD equations, which are not present in the random-phase models.

\section{Dropout Patterns at $r=1 \mathrm{AU}$}

We examine the dropout patterns at $r=1$ AU from the Sun in terms of particle trajectories over different time ranges. For greater clarity and generality, we express the time ranges in terms of the pathlength, $s=v t+0.1 \mathrm{AU}$, where $v$ is the particle speed (which is constant in a static magnetic field, as used in our simulations). Since we inject particles at $t=0$ and $r=0.1 \mathrm{AU}, v t$ expresses 

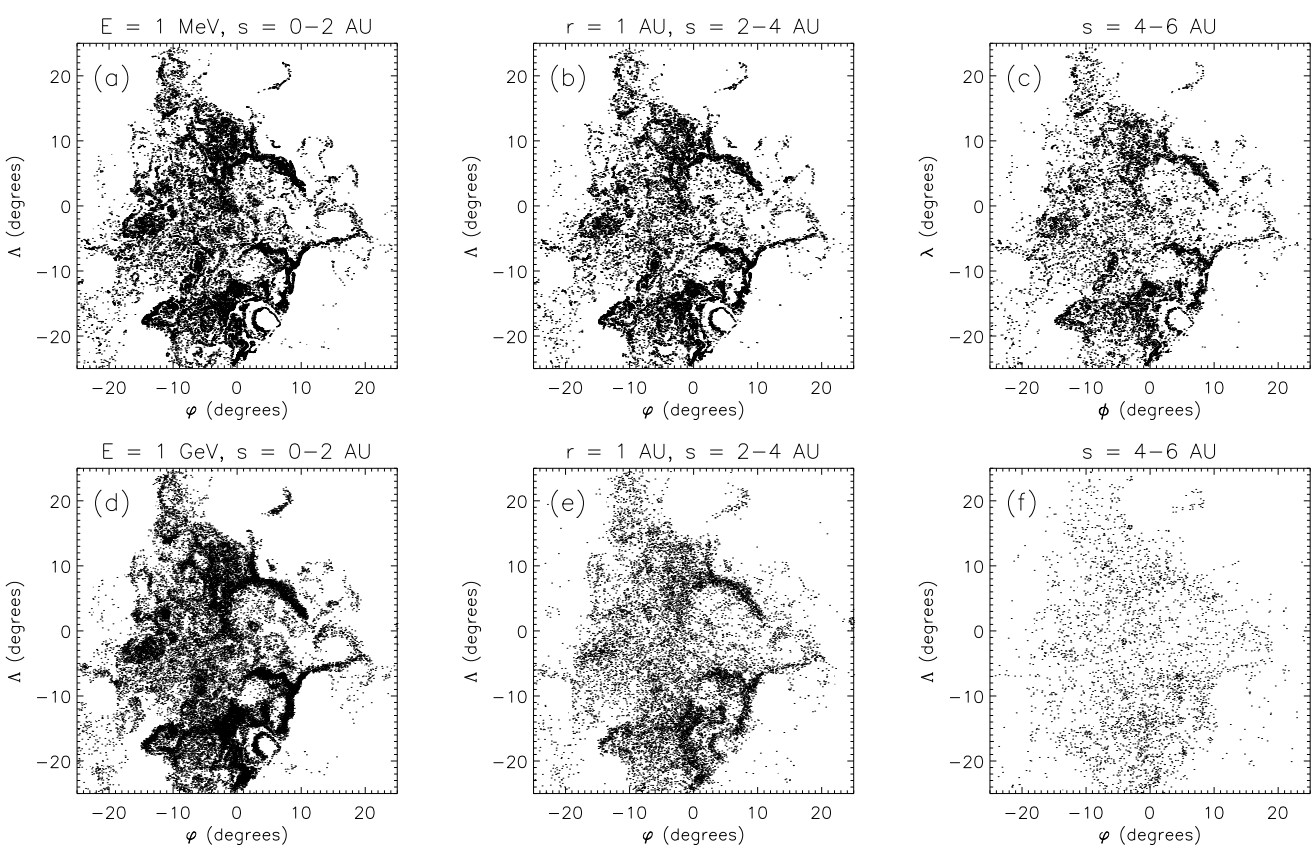

Figure 2: Heliolongitude and heliolatitude when crossing $r=1$ AU of 50,000 solar energetic protons that started at random initial locations at $r=0.1 \mathrm{AU}$ within a circle of radius $2.5^{\circ}$, for the 2D MHD + slab magnetic fluctuation model. We show proton arrival locations at $r=1 \mathrm{AU}$ for various ranges of time, expressed in terms of pathlength from the Sun, $s=v t+0.1 \mathrm{AU}$, for kinetic energy $E=1 \mathrm{MeV}$ (a-c) and $E=1 \mathrm{GeV}(\mathrm{d}-\mathrm{f})$.

the pathlength along the actual particle orbit beyond $r=0.1 \mathrm{AU}$, and we add $0.1 \mathrm{AU}$ to account for the pathlength before injection (assuming radial propagation there).

For low-energy protons of $1 \mathrm{MeV}$, Figure 2(a) shows that the initial dropout pattern of arrival locations during $s=0$ to 2 AU almost exactly matches the pattern of field line locations at $r=1$ AU (Figure 1(e)). In other simulations, we have found that this holds up to a proton energy of $100 \mathrm{MeV}$. Thus for early times we confirm that we can learn about dropouts in particle trajectories by examining the distribution of magnetic connectivity in terms of field line trajectories. At this low energy, Figures 2(b) and (c) show quite similar distributions at later times, corresponding to $s$ ranges from 2 to $4 \mathrm{AU}$ and 4 to $6 \mathrm{AU}$, respectively. The protons arriving at these later times have typically traveled beyond $r=1 \mathrm{AU}$ and scattered back, so our results indicate that during outward transport and backscattering the low energy protons remain quite close to their initial field lines.

Figure 2(d) shows that at $E=1 \mathrm{GeV}$, the proton arrival locations at early times (during $s=0$ to $2 \mathrm{AU}$ ) have spread slightly compared with the field line locations. The distribution for $s=2$ to 4 AU (Figure 2(e)) has spread noticeably over small scales. Then for $s=4$ to 6 AU (Figure 2(f)), the whole dropout pattern is mostly washed out, indicating substantial cross-field transport during transport forward and backward along the interplanetary magnetic field. Nevertheless, there is still a clear outer boundary to the distribution of arrival locations, which remains rather sharp. 

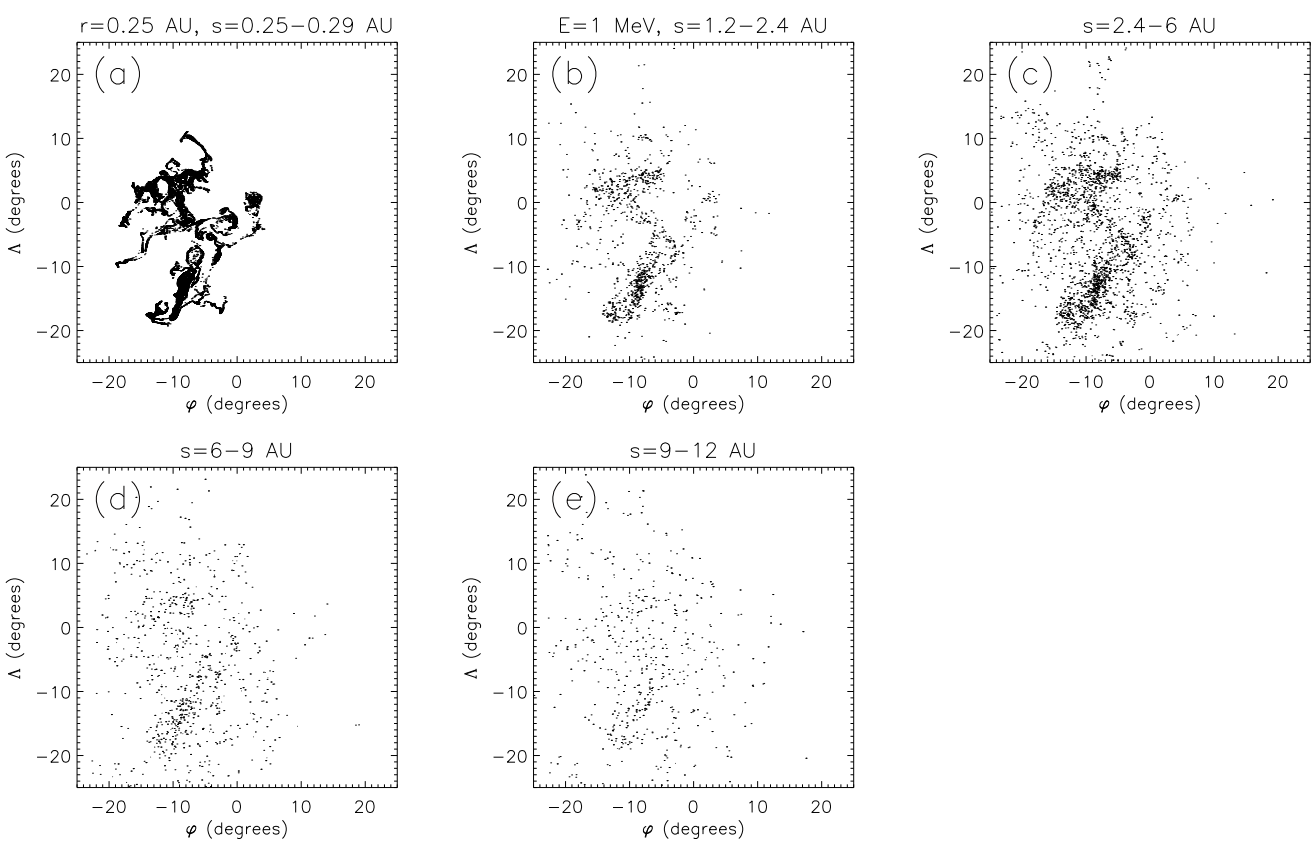

Figure 3: Heliolongitude and heliolatitude when crossing $r=0.25 \mathrm{AU}$ of 50,000 solar energetic protons at $E=1 \mathrm{MeV}$ that started at random initial locations at $r=0.1 \mathrm{AU}$ within a circle of radius $2.5^{\circ}$, for the 2D MHD + slab magnetic fluctuation model, for various ranges of time, expressed in terms of the pathlength $s$. As in Figure 2, the broader distribution at late times has a rather sharp edge and is still not fully diffusive. This Figure indicates the type of SEP distributions that could be observed by the Solar Probe Plus and Solar Orbiter missions when they approach close to the Sun; time intensity profiles could be very different from event to event, depending on the spacecraft location relative to the dropout structure.

\section{Implications for Solar Probe Plus and Solar Orbiter Observations}

In this Section we explore some interesting properties of the lateral distribution of SEPs from impulsive solar events when observed close to the Sun, and the prospects for observing such features with the upcoming Solar Orbiter and Solar Probe Plus missions. As seen in Figure 3(a), protons in the initial pulse had an angular distribution exactly like the magnetic field line distribution (Figure 1(c)), indicating transport along the turbulent field lines. After backscattering from larger $r$, the angular distribution of protons arriving at $r=0.25$ AU during $s=1.2$ to $2.4 \mathrm{AU}$ (Figure 3(b)) is similar to the field line distribution but now somewhat spread out, indicating some cross-field transport. Later, during $s=2.4$ to $9 \mathrm{AU}$ (Figure 3(c-d)), there is a superposition of that distribution, with minor spreading from the field line distribution, together with a broader distribution. Then from $s=9$ to 12 AU (Figure 3(e)) only the broader distribution is apparent. Note, however, that the broader distribution is still confined to a specific angular region, with a rather sharp edge. This region corresponds well with the envelope of the field line distribution at $r=0.5$ AU (Figure 1(d)), which in this representation is predominantly at $\varphi<0$. Thus the effect of cross-field is limited by trapping boundaries, and the spread of the particle distribution at fixed radius at these times is not well described as a diffusive process over the entire sphere. 
In general, a moving spacecraft may measure a combination of effects of parallel transport (governing the time-intensity profile along a field line) and the lateral distribution (manifest as dropout features). For a spacecraft near Earth, the time scales are well separated, with parallel transport effects taking place over longer time scales than dropouts, so both processes can be studied. However, as seen in Figure 3, at $r=0.25$ AU the particle intensity along a given field line varies rapidly during the initial pulse, which is also the time period when the dropout pattern is clearest. This may make it challenging to distinguish between changes due to parallel transport or due to dropouts, unless repeated dropouts are observed. A further issue is that solar events may involve multiple bursts of particle acceleration and release. These are often indistinguishable after transport to $1 \mathrm{AU}$, but may represent an additional source of time structure in observations close to the Sun.

The Solar Orbiter mission aims to reach an orbit with perihelion at about $r=0.28 \mathrm{AU}$, where it may have an angular speed close to the Sun's rotational speed. This is useful for extended observations of specific regions on the solar surface. For dropouts, the implication is that Solar Orbiter may be nearly co-rotating with the filamentary field line distribution. This will minimize the effect of the dropout pattern and make the SEP time profiles sensitive mainly to injection and parallel transport effects.

When a spacecraft nearly co-rotates with the Sun, or if the time required to traverse dropout features is longer than their duration, the time intensity profile will be very different from event to event, depending on the spacecraft location relative to the dropout structure. At $r=0.25 \mathrm{AU}$, Figure 3 shows that even if the angular distance from the mean field connection is small, a spacecraft that remains in a region devoid of connected field lines may see no initial pulse of SEPs, only those backscattering with cross-field transport from greater $r$. They should then detect a Sunward anisotropy of SEPs. On the other hand, a spacecraft in a well-connected region should see a sharp coherent pulse of nearly scatter-free SEPs followed (possibly after a gap) by the wake of backscattered SEPs.

The Solar Probe Plus mission aims to reach an orbit with a much closer perihelion at $r=0.04$ $\mathrm{AU}$, where the orbital speed will approach $200 \mathrm{~km} \mathrm{~s}^{-1}$, which would correspond to an angular speed of $3.4 \times 10^{-5} \mathrm{~s}^{-1}$. This will be faster than the solar rotation frequency by $3.1 \times 10^{-5} \mathrm{~s}^{-1}$, and Solar Probe Plus would traverse the field line structures at $6.4^{\circ} \mathrm{h}^{-1}$. This close to the Sun, the SEPs observed by the EPI-Hi and EPI-Lo instruments [16] should be essentially scatter-free, so the time profile of SEPs along connected field lines should reflect the time dependence of injection by the flare, which could last for tens of minutes. Dropout features should be very sharp (see, e.g., Figure 1(b), for a distance of 0.05 AU from the source) and could quite possibly be observed during the course of SEP emission. Simultaneous information about the time profile and location of particle injection (e.g., from $\gamma$-ray imaging) would provide valuable data concerning magnetic connectivity near the Sun.

Considering observations when Solar Probe Plus is farther from the Sun, the spacecraft orbit as described above would co-rotate with the Sun at $r=0.13 \mathrm{AU}$, and at $r=0.25 \mathrm{AU}$ its orbital angular frequency will be about 4 times lower than the Sun's rotation. With a relative angular speed of $2.1 \times 10^{-6} \mathrm{~s}^{-1}$ or about $0.4^{\circ} \mathrm{h}^{-1}$, it will be more difficult to measure dropouts during the initial pulse of SEPs. At larger distances, the duration of the dropout pattern will increase and dropouts should again be readily detectable. 


\section{Acknowledgments}

This work was supported by grants BRG5880009 and RTA5980003 from the Thailand Research Fund, the US National Science Foundation Solar Terrestrial program grant AGS-1063439, and NASA grants NNX14AI63G (Heliospheric Grand Challenge Research) and NNX15AB88G (Living with a Star Program). WHM is a co-investigator on the ISIS instrument suite (EPI-Hi and EPI-Lo) and is partially supported by the Solar Probe Plus project.

\section{References}

[1] Belcher, J. W., \& Davis, L., Jr. 1971, JGR, 76, 3534

[2] Bieber, J. W., Matthaeus, W. H., Smith, C. W., et al. 1994, ApJ, 420, 294

[3] Bieber, J. W., Wanner, W., \& Matthaeus, W. H. 1996, JGR, 101, 2511

[4] Cash, J. R., \& Karp, A. H. 1990, ACM Trans. Math. Softw., 16, 201

[5] Chuychai, P., Ruffolo, D., Matthaeus, W. H., \& Meechai, J. 2007, ApJ, 659, 1761

[6] Chuychai, P., Ruffolo, D., Matthaeus, W. H., \& Rowlands, G. 2005, ApJL, 633, L49

[7] Dalena, S., Chuychai, P., Mace, R. L., et al. 2012, CoPhC, 183, 9

[8] Giacalone, J., Jokipii, J. R., \& Matthaeus, W. H. 2006, ApJL, 641, L61

[9] Giacalone, J., Jokipii, J. R., \& Mazur, J. E. 2000, ApJL, 532, L75

[10] Gosling, J. T., Skoug, R. M., McComas, D. J., \& Mazur, J. E. 2004, ApJ, 614, 412

[11] Holmes, S. A., \& Featherstone, W. E. 2002, JGeod, 76, 279

[12] Isaacs, J. J., Tessein, J. A., \& Matthaeus, W. H. 2015, JGRA, 120, doi:10.1002/2014JA020661

[13] Matthaeus, W. H., Goldstein, M. L., \& Roberts, D. A. 1990, JGR, 95, 20673

[14] Matthaeus, W. H., Gray, P. C., Pontius, D. H., Jr., \& Bieber, J. W. 1995, PhRvL, 75, 2136

[15] Mazur, J. E., Mason, G. M., Dwyer, J. R., et al. 2000, ApJL, 532, L79

[16] McComas, D. J., Alexander, N., Angold, N., et al. 2014, SSRv, doi:10.1007/s11214-014-0059-1

[17] Parker, E. N. 1958, ApJ, 128, 664

[18] Press, W. H., Teukolsky, S. A., Vetterling, W. T., \& Flannery, B. P. 1992, Numerical recipes in FORTRAN: The art of scientific computing, 2nd ed. (Cambridge: Cambridge Univ. Press), 713

[19] Reames, D. V. 1999, SSRv, 90, 413

[20] Ruffolo, D., \& Khumlumlert, T. 1995, GeoRL, 22, 2073

[21] Ruffolo, D., Matthaeus, W. H., \& Chuychai, P. 2003, ApJL, 597, L169

[22] Ruffolo, D., Seripienlert, A., Tooprakai, P., Chuychai, P., \& Matthaeus, W. H. 2013, ApJ, 779, 74

[23] Seripienlert, A., Ruffolo, D., Matthaeus, W. H., \& Chuychai, P. 2010, ApJ, 711, 980

[24] Tessein, J. A., Ruffolo, D., Matthaeus, W. H., \& Wan, M. 2016, GeoRL, 43, 3620

[25] Tooprakai, P., Chuychai, P., Minnie, J., et al. 2007, GeoRL, 34, L17105

[26] Tooprakai, P., Seripienlert, A., Ruffolo, D., Chuychai, P., and Matthaeus, W. H. 2016, ApJ, 831, 195

[27] Trenchi, L., Bruno, R., Telloni, D., et al. 2013, ApJ, 770, 11

[28] Zimbardo, G., Pommois, P., \& Veltri, P. 2004, JGR, 109, A02113 